\title{
Alpha-MSH: Could this anti-inflammatory mediator be the culprit in Parkinson's Disease?
}

\author{
Jay S. Dela Cruz, Ph.D. \\ InTouch BioSolutions LLC, Moreno Valley, CA 92557 USA; E-mail: jdelacruz@intouchbio.com; Phone: (951) 330-5482
}

\begin{abstract}
We now show that key processes implicated in synuclein pathogenesis such as impairment of cellular autophagy and alphasynuclein ( $\alpha$-syn) aggregation are induced by alpha-melanocyte stimulating hormone (alpha-MSH), a microglia-secreted antiinflammatory mediator. We employed the pigmented melanoma cell line MNT-1 as a novel in-vitro cell model of melanincontaining dopaminergic neurons (DNs) of the substantia nigra (SN). Melanin levels serve as a reliable readout of autophagy in MNT-1 and exposure to alpha-MSH resulted in a decrease in melanin and a failure to slow down glucose consumption, which induced cell death by apoptosis. ASIP (agouti-signaling protein), the natural biologic inhibitor of alpha-MSH, blocked and reversed the effects of alpha-MSH. Mice administered intranasal alpha-MSH exhibited progressive decline in gait, a prevalent condition seen in patients with Parkinson's Disease (PD). Moreover, we observed what may be $\alpha$-syn aggregation in the SN pars compacta (SNpc). SNpc and striatal tyrosine hydroxylase (TH) density showed very modest reduction in this animal PD model consistent with PD pathology at the very early stage of disease.
\end{abstract}

\section{Fundamental Questions Addressed by this Study}

1. What may be the pathophysiologic link between neuroinflammation and impairment of autophagy in PD?

2. What may be a microglia-derived mediator that impairs autophagy and induces alpha-synuclein aggregation in dopaminergic neurons in PD?

3. What drives neurotoxicity that could lead to oxidative stress and ROS-mediated cell loss in PD?

Keywords: Parkinson's disease, alpha-MSH, melanocyte stimulating hormone, alpha-synuclein, autophagy, melanoma, PD model, MNT-1, MC1R, microglia, neuroinflammation, etiology, substantia nigra, dopaminergic neurons, ASIP agoutisignalling protein

\section{Introduction}

Neuroinflammation is primarily mediated by activated microglia, which may play a central role in PD induction and progression. Microglia are present at high density in the SN as compared to adjacent regions (Wang 2015) and are observed to be present prior to development of synuclein pathology (Olanow 2019). Indeed, injection of lipopolysaccharide (LPS) within the SN resulted in microglia activation and subsequent formation of synuclein inclusions in DNs and eventual cell loss (Gao 2008; Castano 2002). The exact mechanism by which activated microglial cells may promote $\alpha$-syn aggregation and neurotoxicity remains unclear.
Autophagy is a cellular response to environmental stress (e.g., low glucose) resulting in decreased metabolism and increased recycling of cellular components by lysosomal degradation. Autophagy impairment is observed in PD pathology and gives rise to accumulation and aggregation of cellular debris and proteins, including $\alpha$-syn (e.g., Lewy bodies and inclusions). Indeed, post-mortem histology of PD SN revealed autophagic degeneration and reduction in key markers of autophagy including, LAMP-1 (Chu 2014). Moreover, mice with DNs deficient in autophagy developed synuclein inclusions, neuronal cell loss and motor deficits (Sato 2018). Consistent with these findings is the observation that genes associated with PD (e.g., SNCA, GBA and LRRK2) play key roles in cellular autophagy (Abeliovich 2016). 
The connection between neuroinflammation and impairment of autophagy in PD pathology is not clear. One hypothesis is that activated microglia-derived factor(s) are released and persist with chronic inflammation and is responsible for the impaired autophagy phenotype and synuclein pathology observed in PD. We now provide evidence in support of this claim.

The 13 a.a. peptide alpha-MSH is an anti-inflammatory mediator released by activated microglial cells (Delgado 1998, Catania 2000) and whose receptor (e.g., MC1R) is expressed by nigral neurons (our results and Chen 2017). Alpha-MSH is implicated in synuclein diseases and found elevated in the cerebrospinal fluid of patients with PD and multiple system atrophy (MSA) (Rainero 1988; Konagaya 1991). Moreover, elevated levels are associated with negative consequences in PD and pharmacologic reduction results in improvements in symptoms (Barbeau 1976; Valverde 1995; Anton-Tay 1971). In addition, beta-MSH (similar in function as alpha-MSH) aggravated symptoms when administered to PD patients (Cotzias 1967), presumably by reducing striatal dopamine levels (Torre 1986).

To gain insight into the role of alpha-MSH in synuclein pathology, we employed a human melanoma cell line MNT-1 (Cuomo 1991) as a model of SN DNs. We chose this system because of its similarity to the neurons affected in PD; both SN DNs and melanoma cells are pigmented and express $\alpha$ syn. Moreover, a clear association between melanoma and PD incidence (Bertoni 2010; Bose 2018) suggested the possibility of a shared etiology involving the loss of autophagy in malignancy (Nassour 2019) and synuclein pathogenesis. Melanin levels correlate with activation of autophagy in MNT-1 (Kalie 2013). This is consistent with the observation that decreased neuromelanin in PD coincided with "autophagic degeneration" or increased number of vacuoles with cytoplasmic materials in melanized neurons of the SN in PD patients (Anglade 1997). Finally, both melanoma cells and SN DNs share a common embryonic origin, express the melanocortin receptor for alpha-MSH, MC1R; and more importantly, produce dopamine (McEwan 1987).

\section{Results}

We observed the hallmark features of synuclein pathology in MNT-1 cells exposed to alpha-MSH in a manner that was reproducible, robust and specific. Alpha-MSH disabled cellular autophagy so that melanin production was significantly reduced (Figure 1). Brief exposure to alpha-MSH was sufficient to block cellular autophagy, which was reversed by treatment with ASIP, the natural biologic inhibitor of alpha-MSH (Suzuki 1997, Abdel-Malek 2001) (Figure 2). As an inverse agonist, ASIP can engage the MC1R receptor and induce the opposite response, thereby, effectively reversing the effects of alpha-MSH (Abdel-Malek 2001; Sakai 1997; Sviderskaya 2001). Alpha-MSH treated MNT-1 cells exhibited impaired autophagy by failing to slow down nutrient consumption so that glucose levels in the culture declined faster than with untreated cells (Figure 3a). Consequently, exposure to alpha-MSH resulted in cell death by apoptosis, which was inhibited by ASIP (Figure 3b-c). Finally, alpha-MSH induced accumulation and aggregation of $\alpha$-syn in MNT-1, which was blocked by ASIP (Figure 4).

The MC1R receptor for alpha-MSH is expressed in human SN DNs (Figure 5), non-human primates (our data, not shown) and mice (Chen 2017). In light of our findings in-vitro, we hypothesize that intranasal delivery of alpha-MSH to the brain of mice will result in a) synuclein pathology in the SN DNs and b) progressive decline in gait performance. Indeed, we observed what may be $\alpha$-syn aggregation in the SNpc in a mouse administered alpha-MSH (Figure 6), which also exhibited progressive gait imbalance (Figure 7, video link to PPT file: [External Data], Table I). Reduction in SNpc TH+ neurons and striatal $\mathrm{TH}+$ density were minimal (Figure 8).

\section{Discussion}

Presence of activated microglial cells is a hallmark feature of PD pathology. Our findings suggest that an antiinflammatory hormone, alpha-MSH, released by activated microglial cells may be the central mediator that triggers disease induction and drive disease progression in PD (Figure 9). We propose that exposure of MC1R-expressing (Figure 5) bystander DNs to abnormal levels of alpha-MSH triggers impairment of cellular autophagy (Figure 1-3, also observed in Kim 2013) resulting in accumulation and aggregation of proteins including $\alpha$-syn (Figure 4 and Figure 6). Sustained exposure may not be necessary to induce a block in autophagy, which may be reversed upon treatment with ASIP (Figure 2).

Alpha-MSH is a regulator of energy homeostasis and cellular metabolism (Anderson 2016). As an anorexigenic hormone, it is a signal to cells that there is adequate levels of nutrients (e.g., glucose) around. We hypothesize that SN DNs exposed to abnormal levels of alpha-MSH maintain a constant metabolic rate even under low nutrient conditions (Figure 3), thereby further depleting an already low nutrient 
environment. Therefore, we propose that cells in which autophagy is severely impaired (e.g., marked by presence of Lewy bodies or $\alpha$-syn inclusions) will outcompete normal or less affected cells for available nutrients and outlive them. Nutrient (glucose) deprivation drives neurotoxicity in these vulnerable cells that ultimately succumb to oxidative stress and elevated reactive oxygen species (ROS). [Indeed, high levels of ROS and decreased GSH (glutathione) levels are observed in the SN in PD (Fahn 1992; Wypijewska 2010; Pearce 1997), a pathology also observed in tissues that experience glucose deprivation (Marambio 2010)]. Dying neurons trigger another round of microglia activation, which release alpha-MSH and synuclein pathology is propagated. After many rounds, gradual yet significant cell loss manifests in a synucleinopathy such as PD (Figure 9).

Consistent with $\alpha$-syn accumulation and aggregation observed when MNT-1 was exposed to alpha-MSH (Figure 4), we observed what may be $\alpha$-syn aggregation in the $\mathrm{SN}$ in a mouse administered alpha-MSH by intranasal route (Figure 6). The same mouse and two other mice administered alphaMSH developed gait imbalance that progressively worsened with time, as revealed by a modified rotarod test (Figure 7, video link to PPT file: [External Data], Table I). Evaluation of $\mathrm{SNpC} \mathrm{TH}+$ neuron and striatal $\mathrm{TH}+$ density revealed a very modest reduction in these mice (Figure 8 ), suggesting a disease state that is consistent with very early stage PD. Indeed, patients who were recently diagnosed ( $<3$ years) also showed modest PD pathology (Kordower 2013). Therefore, extending the period of time post alpha-MSH administration (> 2 months, e.g., 6 or 9 months) is expected to exacerbate synuclein pathology and gait performance. If true, the progressive nature of this animal PD model will permit adequate time for therapeutic intervention and testing of potential disease-modifying drugs.

\section{Conclusion}

We propose that alpha-MSH plays a central role in pathogenesis and progression in PD. And to pursue strategies that target alpha-MSH and related pathways, evidence is required in higher species that shows abnormal exposure to alpha-MSH is sufficient to induce synuclein disease. Nonhuman primates (NHPs) is the preferred species model since nigral neurons, as in humans, are more heavily melanized relative to rodents (Emborg 2007). Moreover, human and NHP alpha-MSH and receptors are identical or nearly identical. A successful demonstration of the use of alphaMSH to induce synucleinopathy in NHP offers the investigator a novel pathophysiologically relevant, cost-effective, technically simple and non-invasive model to evaluate new therapies against PD and other synucleinopathies (e.g., MSA and DLB). Our in-vitro DN cell model (MNT-1 + alpha-MSH) offers a platform on which to screen for a novel therapeutic that reverses impairment of autophagy and blocks $\alpha$-syn accumulation and aggregation.

\section{Materials and Methods}

Growth/culture conditions: MNT-1 was a gift from Dr. Michael D. Marks (UPenn). MNT-1 growth media reagents were purchased from Thermofisher.com : DMEM (11995065) supplemented with 15\% FBS (10437-028), 10\% MEM NEAA (1037-021), 10\% AIM V (31035-025) and pen/strep (from 100X, 15140-122). Cells were maintained in $25 \mathrm{~cm}^{2}$ canted neck tissue culture flask (353109) in $4 \mathrm{ml}$ of media in a humidified incubator and $5 \% \mathrm{CO}_{2}$. Passaging was performed, once confluency reached $90 \%$, by trypsinization $(0.25 \%$ Tyrpsin, 15050-057) and neutralization in growth media. Cells were diluted 1:3 or 1:6 depending on need and seeded in $4 \mathrm{ml}$ growth media. Growth media was replaced every 2-3 days. When required for cell assays, confluency was $80-90 \%$ when cells were trypsinized and washed. Trypsinized cells were washed 1Xs in growth media and resuspended in the appropriate assay buffer. Cell counts were performed in trypan blue using a hemacytometer under a microscope. Alpha-MSH (GenScript, RP10644) was prepared in artificial cerebrospinal fluid (aCSF, Tocris, 3525) and endotoxin removed $(<0.01 \mathrm{EU} / \mathrm{ml}$, Pierce, 88274). Recombinant human (rh)ASIP (R\&D Systems, 9094-AG) was prepared in aCSF (Tocris).

Visual observation of melanin reduction in alpha-MSH treated MNT-1 cells: 20,000 MNT-1 cells were seeded per well in a 24-well flat bottom tissue culture plate (Costar 353047) in 500 microliter of growth media and the plate placed in a humidified incubator with $5 \% \mathrm{CO}_{2}$. After 48 hours, 250 microliter of supernatant was removed and replaced with the same volume of growth media or growth media containing $200 \mathrm{nM}$ alpha-MSH (final=100nM). After 7 days, the plate was placed under ambient light and a photograph taken using a camera.

Alpha-MSH reduces cellular autophagy activation by Rapamycin: 10,000 MNT-1 cells were seeded per well in a 96well flat bottom tissue culture plate (Costar 3596) in 100 microliter of growth media and placed in a humidified incubator with $5 \% \mathrm{CO}_{2}$. After 48 hours, $100 \mathrm{nM}$ Rapamycin 
(AG Scientific, R1018) alone or mixed with 100nM alpha-MSH or assay buffer was added to the cells in triplicate wells in a final volume of 200 microliter. After 7 days, melanin levels were determined in each well as described below.

Measurement of melanin in 96-well plate format: A) Conventional method: Supernatant from wells containing adherent MNT-1 was removed and added 200 microliter of $1 X$ PBS. 1X PBS was removed and the plate dabbed upsidedown and allowed to sit this way over a stack of paper towel for 2 minutes. After another dab, 100 microliter of $1 \mathrm{~N} \mathrm{NaOH}$ was added to each well and the plate moved to a plate shaker (200 rpm, 5 minutes). The plate was moved to $37^{\circ} \mathrm{C}$ incubator (16-18 hours). Samples were further solubilized by resuspension and the absorbance at $405 \mathrm{~nm}$ measured using a plate reader (Molecular Devices Thermomax and Softmax Pro software). B) Novel method using fluorescence (fluorescein) quenching by melanin: Plates frozen overnight in below $-70^{\circ} \mathrm{C}$ were thawed in room temperature for 20 minutes. A fluorescein-based dye was prepared in a cell lysis solution and 200 microliter added to each well and the plate placed on shaker in the dark for 10 minutes. The lysed cells were resuspended and 90 microliter transferred to a whiteopaque 96-well plate (Costar 353296) and fluorescence measured using a fluorescence plate reader (Cytofluor 4000, Gain 50, Ex 485/20, Em 530/25).

Immunofluorescence detection of alpha-synuclein accumulation and aggregation in MNT-1: 8,000 MNT-1 cells were seeded in each well of an 8-chamber glass slide (LabTek, 154941) in 400 microliter growth media and placed in the humidified incubator and $5 \% \mathrm{CO}_{2}$. After 72 hours, supernatant was removed and replaced with an equal volume of growth medium containing $25 \mathrm{nM}$ alpha-MSH, 100nM rhASIP, both or with growth medium alone. After another 48 hours in culture, supernatant is removed by aspiration and 500 microliter of fixiation buffer $(4 \%$ paraformaldehyde in PBS, Biolgend) added to each well and kept in the dark at room temperature. After 5 hours, fixation buffer was removed and 500 microliter of $1 X$ PBS added and removed. $1 / 500$ diluted alpha-synuclein monoclonal antibody (Syn 211, Thermofisher, AHB0261) prepared in intracellular staining buffer (ISB, 0.25\% Triton-X 100, 5\% goat serum in $1 \mathrm{X}$ PBS) was added in 400 microliter per well and the slide place in $4^{\circ} \mathrm{C}$ refrigerator. After two washes with ISB, 400 microliter of $1 / 1000$ diluted anti-mouse IgG-Alexafluor 488 (Biolegend, 405319) was added and the slide returned to $4^{\circ} \mathrm{C}$ refrigerator. After 2 washes with ISB and 1 wash with 1 X PBS, the slide was mounted with ProLong ${ }^{\circledR}$ Gold antifade reagent and coverslip placed. The slides were viewed (140X magnification) using a fluorescence microscope (Olympus
BH2-RFCA with BP490 cube for blue wavelengths) and images were captured using a Zeiss AxioCam MRm camera.

Detection of apoptosis by flow cytometry: MNT-1 cells were seeded in 6-well tissue plate (Costar 353046) and added control buffer or 50nM alpha-MSH in $2 \mathrm{ml}$ of growth media in a humidified incubator with $5 \% \mathrm{CO}_{2}$. After 4,7 (seeded at 40,000 cells on Day 0 ) and 9 days (seeded at 20,000 cells in $25 \%$ growth media in DMEM on Day 0$), 0.9 \mathrm{ml}$ of supernantant was harvested and added 100 microliter of the apoptosis reagent (CellEvent ${ }^{\mathrm{TM}}$ Caspase-3/7 Green Detection Reagent, Thermofisher, C10723) and returned to respective wells for another 30 minutes of incubation. Wells were washed 2Xs with 1X PBS and the cells trypsinized, washed with RPMI $1640+10 \%$ FBS and centrifuged 1800rpm for 7 minutes at room temperature using Sorvall RT7. Cells were resuspended in $1 \mathrm{X}$ PBS and analyzed by flow cytometry (BD FACS Calibur and CellQuest Pro analysis software).

Measurement of cell viability in 96-well plate format: At the end of the incubation period, supernatant from wells containing adherent MNT-1 was removed and added 200 microliter of $1 \mathrm{X}$ PBS. 1X PBS was removed and the plate dabbed upside-down and allowed to sit this way over a stack of paper towels for 2 minutes. After another dab, 100 microliter of CellTiter $96^{\circledR}$ AQueous One Solution Cell Proliferation Assay solution (MTS, Promega G3580) prepared in DMEM + 5\% FBS was added to each well and the plate returned to a humidified incubator and $5 \% \mathrm{CO}_{2}$ with the lid off and allowed develop. The absorbance at 490nm and $650 \mathrm{~nm}$ (background absorbance) was measured using a plate reader (Molecular Devices Thermomax and Softmax Pro software).

Detection of MC1R in human SN: Tissue Processing: All brains were processed as described (Chu et.al., 2006). Briefly, each brain was cut into $2 \mathrm{~cm}$ coronal slabs and then hemisected. The slabs were fixed in $4 \%$ paraformaldehyde for 5 days at $4^{\circ} \mathrm{C}$. After brain blocks were sampled from one side of the brain for pathologic diagnoses, the remaining brain slabs were cryoprotected in $0.1 \mathrm{M}$ phosphate buffered saline (PBS; $\mathrm{pH} 7.4$ ) containing $2 \%$ dimethyl sulfoxide, $10 \%$ glycerol for 48 $\mathrm{h}$ followed by $2 \%$ dimethyl sulfoxide, and $20 \%$ glycerol in PBS for at least 2 days prior to sectioning. The fixed slabs were then cut into 18 adjacent series of $40 \mu \mathrm{m}$ thick sections on a freezing sliding microtome. All sections were collected and stored at $-20^{\circ} \mathrm{C}$ in a cryoprotectant solution prior to processing. Immunohistochemistry: An immunoperoxidase labeling method was used to examine the expressions of MC1R. Endogenous peroxidase was quenched by 20 minutes of incubation in $0.1 \mathrm{M}$ sodium periodate, and background 
staining was blocked by 1 hour of incubation in a solution containing $2 \%$ bovine serum albumin and $5 \%$ normal goat serum. Tissue sections were incubated in anti-MC1R rabbit antibody (1:200; polyclonal, Thermofisher Cat\# PA5-33923) over night at room temperature. After 6 washes, sections were sequentially incubated for 1 hour in biotinylated goat anti-rabbit IgG (1:200; Vector) followed by Elite avidin-biotin complex (1:500; Vector) for 75 minutes. The immunohistochemical reaction was completed with $0.05 \%$ 3,3'-diaminobenzidine and $0.005 \% \mathrm{H} 2 \mathrm{O} 2$. Sections were mounted on gelatin-coated slides, dehydrated through graded alcohol, cleared in xylene, and coverslipped with Cytoseal (Richard-Allan Scientific, Kalamazoo, MI). Immunohistochemical controls: Immunohistochemical control experiments included omission of the primary antibodies (which control for the specificity of the staining procedure and the secondary antibody) and replacement of the primary antibodies with an irrelevant IgG matched for protein concentration. The control sections were processed in a manner identical to that described above. An adsorption control experiment for MC1R antibody was also performed. Briefly, the anti-MC1R rabbit antibody was combined with a fivefold (by weight) of recombinant MC1R peptide (217-232, Alomone Labs) in TBS and incubated at $4{ }^{\circ} \mathrm{C}$ overnight. The immune complexes with the antibody and blocking peptide were centrifuged at $10,000 \mathrm{~g}$ for $20 \mathrm{~min}$. The adsorbed peptide/antibody supernatant was then used in lieu of the primary antibody. This resulted in a total absence of staining (see Figure 5).

Intranasal administration of alpha-MSH: The animal study described was approved by InTouch BioSolutions LLC Institutional Animal Care and Use Committee (IACUC Protocol \# 052017). 16-17 weeks-old C57BL/6J female mice (JAX, 000664) were administered 3.32 ug of alpha-MSH in 10 microliter aCSF $(n=3)$ or aCSF $(n=1)$ every other day for a total of 14 intranasal administrations over 26 days. A Rainin L20 pipette fitted with a tip was used to deliver 10 microliter in the left nostril of toe-pinched confirmed fully anesthetized mouse. After administration, the mouse was allowed to remain in a supine position for 10 minutes under anesthesia and subsequently allowed to fully recover in its respective cage.

Assessment of gait by a modified rotarod: Hind leg gait was assessed using a Rotarod apparatus (IITC Life Science Inc. Series 8) every week for a total of 8 weeks using a modified drum consisting of a polyethylene pipe insulation of $1 \frac{1}{4}$ inch diameter (Grainger, 2CKE8) to encourage gait. A digital video camera was used to record. Mice were allowed to warm-up for 2 minutes at the top speed of 30 RPM. Afterward, the rotarod was quickly restarted and the gait video recorded for another 5 minutes at the top speed of 30 RPM.

Brain perfusion, fixation and staining with anti-alpha synuclein or anti-tyrosine hydroxylase (TH): Brains of anesthetized mice were perfused with $24 \mathrm{ml} 0.9 \% \mathrm{NaCl}$ saline solution. Briefly, the heart was exposed and the descending aorta clamped with a bulldog clamp. Saline was administered via the left ventricle using a 22 G 1-1/2 in needle and the right atrium clipped with a scissor and perfusion terminated after 6 minutes. The brain was carefully removed and placed in 10 $\mathrm{ml}$ of $4 \%$ PFA in $1 X$ PBS solution. After 3 days, the brain was washed $3 \mathrm{Xs}$ with $20 \mathrm{ml}$ of saline and place in $1 \mathrm{X}$ PBS and stored in $4^{\circ} \mathrm{C}$ refrigerator.

Neurohistology Embedding, Sectioning \& Staining performed by Neuroscience Associates, Inc.: Brains were examined, then treated overnight with $20 \%$ glycerol and $2 \%$ dimethylsulfoxide to prevent freeze-artifacts. The specimens were then embedded in a gelatin matrix using MultiBrain ${ }^{\circledR /}$ MultiCord ${ }^{\circledR}$ Technology (NeuroScience Associates, Knoxville, TN). The blocks were rapidly frozen, after curing by immersion in 2-Methylbutane chilled with crushed dry ice and mounted on a freezing stage of an AO 860 sliding microtome. The MultiBrain ${ }^{\circledR} /$ MultiCord $^{\circledR}$ blocks were sectioned in coronally with 35 micrometer $(\mu \mathrm{m})$ setting on the microtome. All sections were cut through the entire length of the specimen segment and collected sequentially into series of 24 containers. All containers contained Antigen Preserve solution (50\% PBS pH7.0, 50\% Ethylene Glycol, 1\% Polyvinyl Pyrrolidone); no sections were discarded. Immunohistochemistry - For immunohistochemistry, free floating sections were stained with desired stain. All incubation solutions from the blocking serum onward used Tris buffered saline (TBS) with Triton X-100 as the vehicle; all rinses were with TBS. After a hydrogen peroxide treatment and blocking serum, the sections were immunostained with (for $\alpha$-syn stain): $1 / 5000$ diluted alpha-synuclein antibody (BD Pharmigen, 610786) or (for tyrosine hydroxylase stain): 1/18,000 diluted anti-TH (Pelfreez, P40101) and incubated overnight at room temperature. Vehicle solutions contained Triton X-100 for permeabilization. Following rinses, (for $\alpha$ syn stain) a 1/1000 diluted biotinylated horse anti-mouse IgG antibody (Vector Laboratories, BA-2001) or (for tyrosine hydroxylase stain) a $1 / 1000$ diluted biotinylated secondary antibody (Vector, BA-1000) was applied. After further rinses Vector Lab's ABC solution (avidin-biotin-HRP complex; details in instruction for VECTASTAIN ${ }^{\circledR}$ Elite ABC, Vector, Burlingame, CA) was applied. The sections were again rinsed, then treated with diaminobenzidine tetrahydrochloride (DAB) and hydrogen peroxide to create a visible reaction product. 
bioRxiv preprint doi: https://doi.org/10.1101/2020.09.27.313957; this version posted September 29, 2020. The copyright holder for this preprint (which was not certified by peer review) is the author/funder. All rights reserved. No reuse allowed without permission.

Following further rinses, the sections were mounted on gelatin coated glass slides, air dried. The slides were dehydrated in alcohols, cleared in xylene and coverslipped. Image capture: The slides were viewed using a microscope (Olympus $\mathrm{BH} 2$ ) and images were captured using a digital camera fitted on the microscope.

\section{Acknowledgements}

I would like to thank my wife, Linh Dela Cruz, who battles PD daily, for her love and support. Our children Hannah, Esther and Joshua have been very helpful and supportive. I am grateful to Dr. Sherie L. Morrison for her invaluable input and support. I am grateful to Dr. Jeffrey H. Kordower for his invaluable input and support. I am thankful for the prayers and support of our families and friends. Thank you, Lord Jesus Christ, for your kindness to me and for guiding me through this work. May all the glory be yours alone.

\section{References}

[1] Abdel-malek ZA, Scott MC, Furumura M, et al. The melanocortin 1 receptor is the principal mediator of the effects of agouti signaling protein on mammalian melanocytes. J Cell Sci. 2001;114(Pt 5):1019-24.

[2] Abeliovich A, Gitler AD. Defects in trafficking bridge Parkinson's disease pathology and genetics. Nature. 2016;539(7628):207-216.

[3] Anderson EJ, Çakir I, Carrington SJ, et al. 60 YEARS OF POMC: Regulation of feeding and energy homeostasis by $\alpha-\mathrm{MSH}$. J Mol Endocrinol. 2016;56(4):T157-74.

[4] Anglade P, Vyas S, Javoy-agid F, et al. Apoptosis and autophagy in nigral neurons of patients with Parkinson's disease. Histol Histopathol. 1997;12(1):25-31.

[5] Antón-tay F, Díaz JL, Fernández-guardiola A. On the effect of melatonin upon human brain. Its possible therapeutic implications. Life Sci I. 1971;10(15):841-50.

[6] Barbeau A, Roy M, Kastin AJ. Double-blind evaluation of oral L-prolyl-Lleucyl-glycine amide in Parkinson's disease. Can Med Assoc J. 1976;114(2):120-2.

[7] Bertoni JM, Arlette JP, Fernandez HH, et al. Increased melanoma risk in Parkinson disease: a prospective clinicopathological study. Arch Neurol. 2010;67(3):347-52.

[8] Bose A, Petsko GA, Eliezer D. Parkinson's Disease and Melanoma: Co-Occurrence and Mechanisms. J Parkinsons Dis. 2018;8(3):385-398.

[9] Castaño A, Herrera AJ, Cano J, Machado A. The degenerative effect of a single intranigral injection of LPS on the dopaminergic system is prevented by dexamethasone, and not mimicked by rh-TNF-alpha, IL-1beta and IFN-gamma. J Neurochem. 2002;81(1):150-7.

[10] Catania A, Airaghi L, Colombo G, Lipton JM. Alphamelanocyte-stimulating hormone in normal human physiology and disease states. Trends Endocrinol Metab. 2000;11(8):304-8.

[11] Chen X, Chen H, Cai W, et al. The melanoma-linked "redhead" MC1R influences dopaminergic neuron survival. Ann Neurol. 2017;81(3):395-406.

[12] Chu Y, Goldman JG, Kelly L, He Y, Waliczek T, Kordower JH. Abnormal alpha-synuclein reduces nigral voltage-dependent anion channel 1 in sporadic and experimental Parkinson's disease. Neurobiol Dis. 2014;69:1-14.

[13] Cotzias GC, Van woert MH, Schiffer LM. Aromatic amino acids and modification of parkinsonism. N Engl J Med. 1967;276(7):374-9.

[14] Cuomo M, Nicotra MR, Apollonj C, Fraioli R, Giacomini P, Natali PG. Production and characterization of the murine monoclonal antibody $2 \mathrm{G} 10$ to a human T4-tyrosinase epitope. J Invest Dermatol. 1991;96(4):446-51.

[15] Delgado R, Carlin A, Airaghi L, et al. Melanocortin peptides inhibit production of proinflammatory cytokines and nitric oxide by activated microglia. J Leukoc Biol. 1998;63(6):740-5

[16] Emborg ME. Nonhuman primate models of Parkinson's disease. ILAR J. 2007;48(4):339-55.

[17] Fahn S, Cohen G. The oxidant stress hypothesis in Parkinson's disease: evidence supporting it. Ann Neurol. 1992;32(6):804-12.

[18] Gao HM, Kotzbauer PT, Uryu K, Leight S, Trojanowski JQ, Lee VM. Neuroinflammation and oxidation/nitration of alphasynuclein linked to dopaminergic neurodegeneration. J Neurosci. 2008;28(30):7687-98.

[19] Kalie E, Razi M, Tooze SA. ULK1 regulates melanin levels in MNT-1 cells independently of mTORC1. PLOS ONE. 2013;8(9):e75313.

[20] Kim ES, Jo YK, Park SJ, et al. ARP101 inhibits $\alpha-\mathrm{MSH}-$ stimulated melanogenesis by regulation of autophagy in melanocytes. FEBS Lett. 2013;587(24):3955-60.

[21] Konagaya $Y$, Konagaya M. [Abnormality of hypothalamic dopaminergic system in neuro-degenerative diseases-evaluation of alpha-melanocyte-stimulating hormone-like immunoreactivity in cerebrospinal fluid]. Rinsho Shinkeigaku. 1991;31(8):821-5.

[22] Kordower JH, Olanow CW, Dodiya HB, et al. Disease duration and the integrity of the nigrostriatal system in Parkinson's disease. Brain. 2013;136(Pt 8):2419-31.

[23] Marambio P, Toro B, Sanhueza C, et al. Glucose deprivation causes oxidative stress and stimulates aggresome formation and autophagy in cultured cardiac myocytes. Biochim Biophys Acta. 2010;1802(6):509-18.

[24] Mcewan M, Parsons PG. Inhibition of melanization in human melanoma cells by a serotonin uptake inhibitor. J Invest Dermatol. 1987;89(1):82-6.

[25] Nassour J, Radford R, Correia A, et al. Autophagic cell death restricts chromosomal instability during replicative crisis. Nature. 2019;565(7741):659-663. 
bioRxiv preprint doi: https://doi.org/10.1101/2020.09.27.313957; this version posted September 29, 2020. The copyright holder for this preprint (which was not certified by peer review) is the author/funder. All rights reserved. No reuse allowed without permission.

[26] Olanow CW, Savolainen M, Chu Y, Halliday GM, Kordower JH Temporal evolution of microglia and $\alpha$-synuclein accumulation following foetal grafting in Parkinson's disease. Brain. 2019;142(6):1690-1700.

[27] Rainero I, Kaye JA, May C, et al. Alpha-melanocytestimulating hormonelike immunoreactivity is increased in cerebrospinal fluid of patients with Parkinson's disease. Arch Neurol. 1988;45(11):1224-7.

[28] Sakai C, Ollmann M, Kobayashi T, et al. Modulation of murine melanocyte function in vitro by agouti signal protein. EMBO J. 1997;16(12):3544-52.

[29] Sato S, Uchihara T, Fukuda T, et al. Loss of autophagy in dopaminergic neurons causes Lewy pathology and motor dysfunction in aged mice. Sci Rep. 2018;8(1):2813.

[30] Suzuki I, Tada A, Ollmann MM, et al. Agouti signaling protein inhibits melanogenesis and the response of human melanocytes to alpha-melanotropin. J Invest Dermatol. 1997;108(6):838-42.

[31] Sviderskaya EV, Hill SP, Balachandar D, Barsh GS, Bennett DC. Agouti signaling protein and other factors modulating differentiation and proliferation of immortal melanoblasts. Dev Dyn. 2001;221(4):373-9.

[32] Torre E, Celis ME. Cholinergic mediation in the ventral tegmental area of alpha-melanotropin induced excessive grooming: changes of the dopamine activity in the nucleus accumbens and caudate putamen. Life Sci.

1988;42(17):1651-7.
[33] Valverde P, Benedito E, Solano F, Oaknin S, Lozano JA, García-borrón JC. Melatonin antagonizes alpha-melanocytestimulating hormone enhancement of melanogenesis in mouse melanoma cells by blocking the hormone-induced accumulation of the c locus tyrosinase. Eur J Biochem. 1995;232(1):257-63.

[34] Wang Q, Oyarzabal E, Wilson B, Qian L, Hong JS. Substance P enhances microglial density in the substantia nigra through neurokinin-1 receptor/NADPH oxidase-mediated chemotaxis in mice. Clin Sci. 2015;129(8):757-67.

[35] Wypijewska A, Galazka-friedman J, Bauminger ER, et al. Iron and reactive oxygen species activity in parkinsonian substantia nigra. Parkinsonism Relat Disord. 2010;16(5):32933. 


\section{Figures}

\section{(A) Rapamycin-induced autophagy}

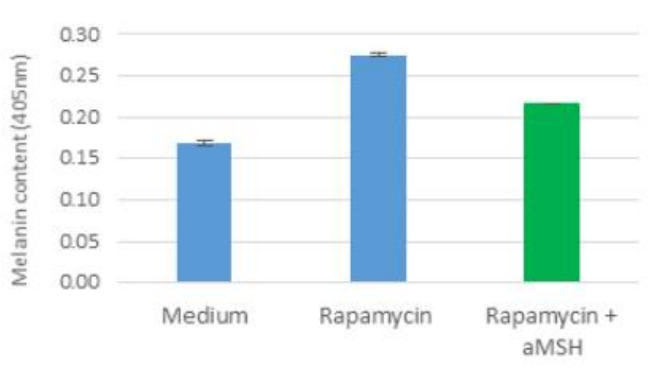

(B) Starvation-induced autophagy

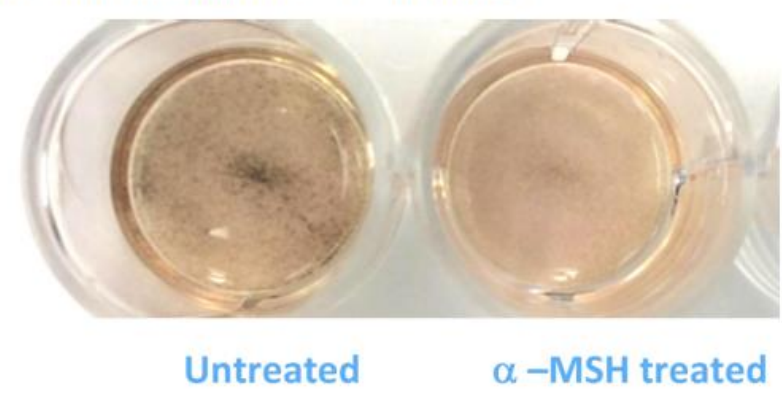

Figure 1: Alpha-MSH disables cellular autophagy. A) Cellular autophagy induced by rapamycin is reduced by alpha-MSH. B) Starvation-induced autophagy is inhibited by alpha-MSH, reducing melanin.

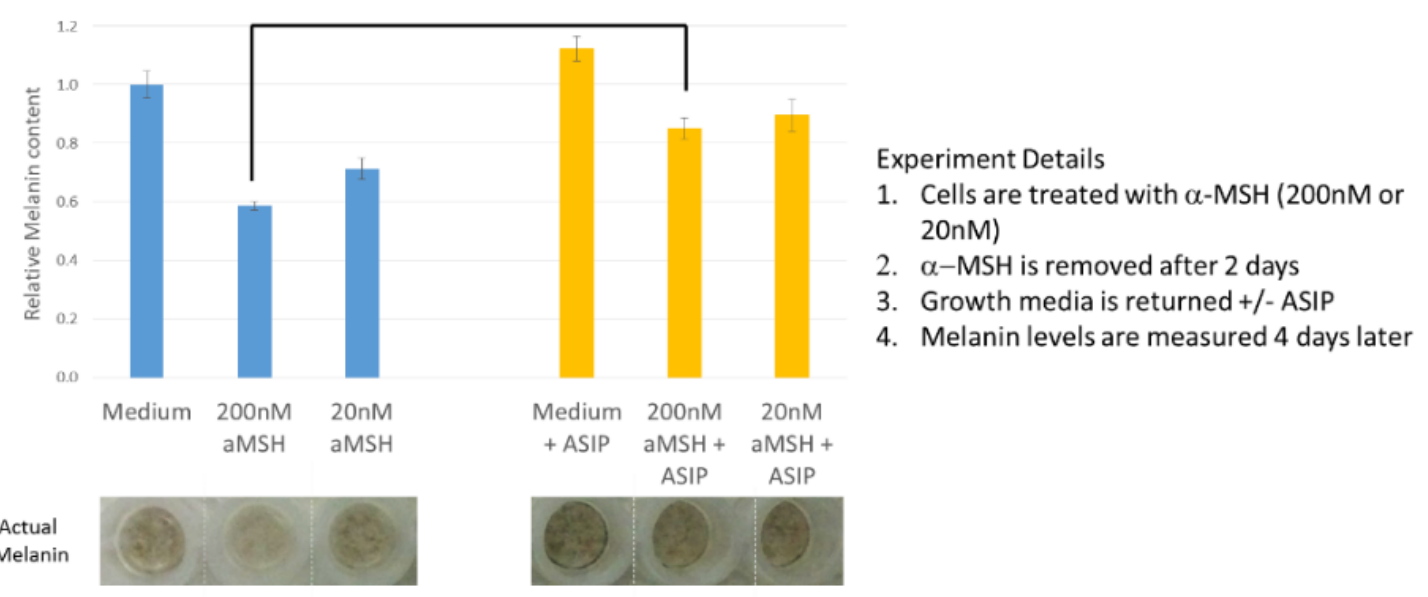

Figure 2: ASIP reverses block in cellular autophagy induced by alpha-MSH. ASIP (acting as inverse agonist) reverses alpha-MSH effects and restores cellular autophagy (starvation). 
(A)



-Untreated - a-MSH
(B)

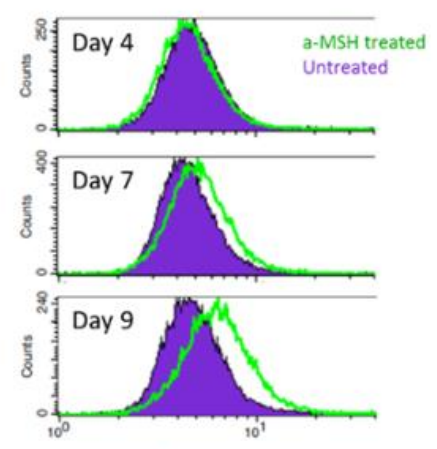

Caspase 3/7 apoptosis detection
(C)

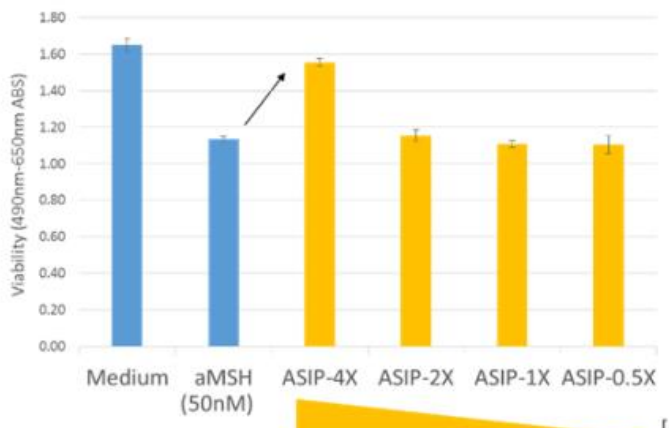

[ASIP]

Experiment Details

aMSH $(50 \mathrm{nM})$

1. Cells are treated with $\alpha-\mathrm{MSH}$ alone

or with varying amounts of ASIP.

2. Cell viability is measured 6 days later

Figure 3: Exposure to alpha-MSH results in cell death by apoptosis; ASIP is protective. A) alpha-MSH treated cells fail to respond to low nutrient conditions and consume glucose abnormally, whereas untreated cells slow down glucose consumption. B) Alpha-MSH treated cells gradually die by apoptosis. C) ASIP blocks alpha-MSH effects and protects against cell death.



Untreated

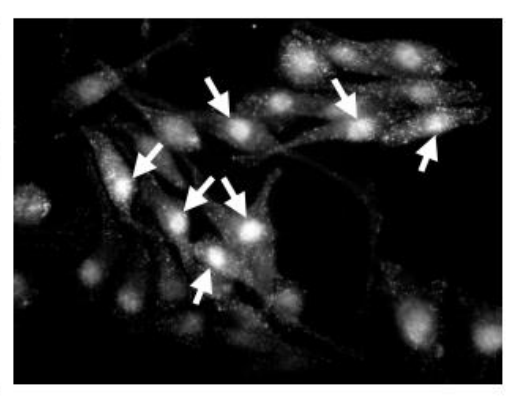

$\alpha-\mathrm{MSH}$ treated

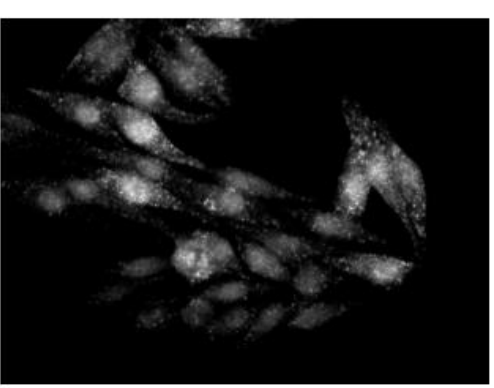

$\alpha-$ MSH treated + ASIP

Figure 4: Alpha-MSH induces accumulation and aggregation of a-syn in MNT-1 cells. ASIP blocks $\alpha-M S H$ induced accumulation and aggregation of a-syn in MNT-1 cells (arrows). 
bioRxiv preprint doi: https://doi.org/10.1101/2020.09.27.313957; this version posted September 29, 2020. The copyright holder for this preprint (which was not certified by peer review) is the author/funder. All rights reserved. No reuse allowed without permission.

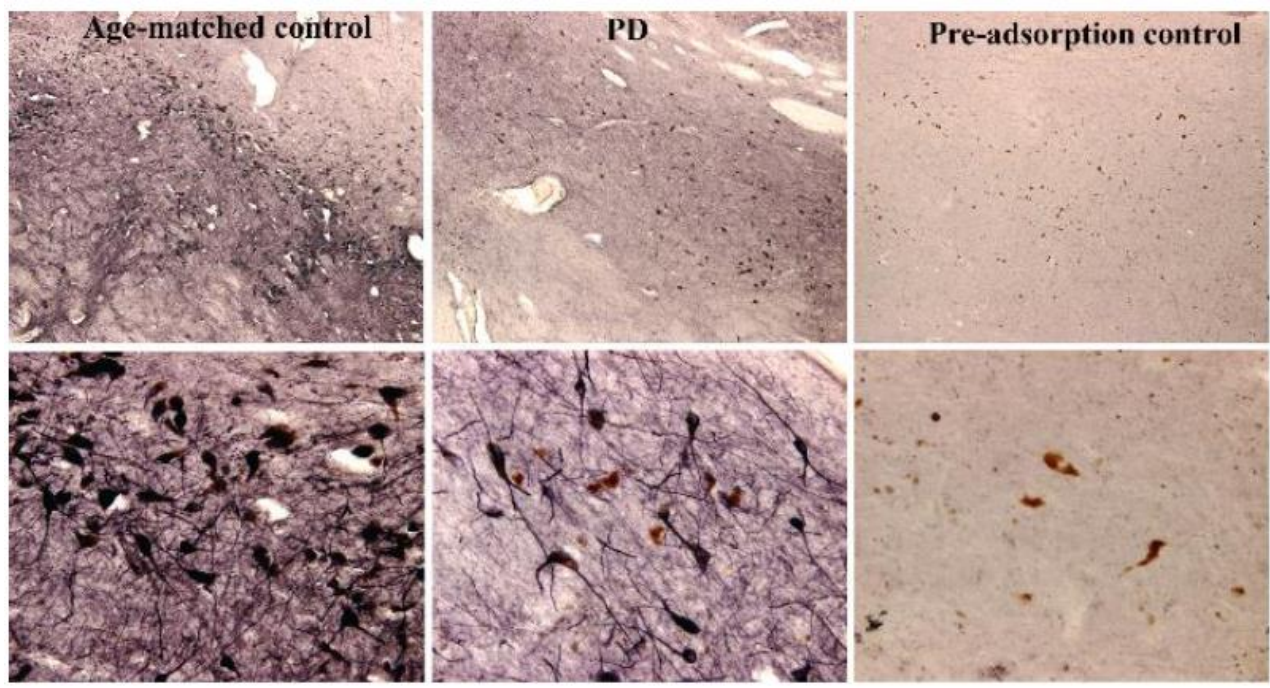

Figure 5: MC1R is expressed in human substantia nigra (SN) dopaminergic neurons. IHC staining of human $\mathrm{SN}$ neurons from age-matched control (left) and PD (center) sections. MC1R staining is confirmed by peptide preadsorbed antibody (right). (Histology credit: Jeffrey H. Kordower, Ph.D. and Yaping Chu, M.D., from Rush University, Chicago)

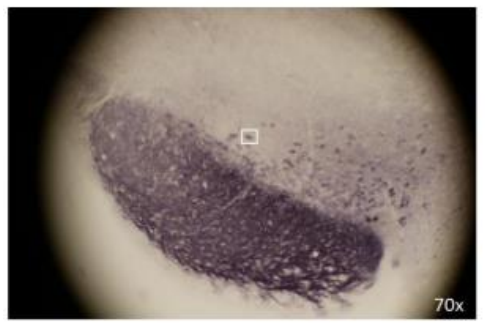

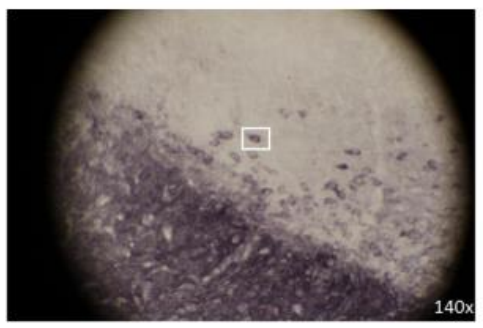

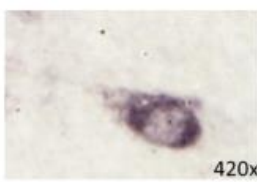

Vehicle control
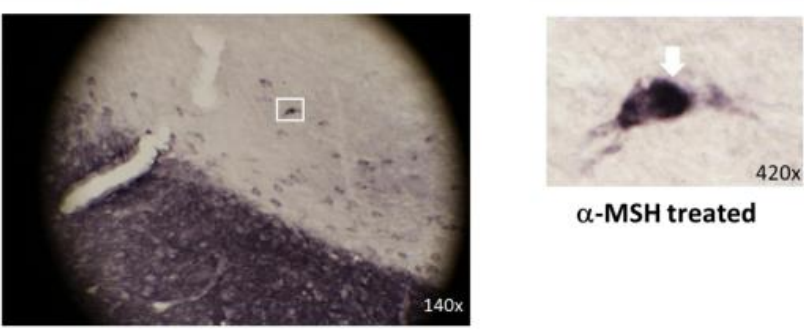

$\alpha-\mathrm{MSH}$ treated

Figure 6: Alpha-MSH treated mouse exhibits what may be a-syn aggregation in the substantia nigra. Ipsilateral SN was examined for presentation of synuclein pathology by IHC. Top panel is SN from vehicle administered (left-nostril) mouse with increasing magnification (left-to-right). Bottom panel is SN from the same mouse shown in Figure 7 administered (left-nostril) alpha-MSH. IHC Credit: Neuroscience Associates, Inc. (NSA) 
bioRxiv preprint doi: https://doi.org/10.1101/2020.09.27.313957; this version posted September 29, 2020. The copyright holder for this preprint (which was not certified by peer review) is the author/funder. All rights reserved. No reuse allowed without permission.


Figure 7: Alpha-MSH treated mice exhibit progressive motor deficit. Alpha-MSH treated (left nostril) mouse \#1 (representative of $n=3$ ) shows progressive decline in gait. No notable impairment was observed 2 weeks (left panel) after 1-month of intranasal. Progressive impairment was observed 4 (right panel), 6 and 8 weeks after (not shown). With impaired right hind leg, loss of symmetry in gait is apparent, center of gravity is shifted inward and left leg moves inward during gait (right panel, circle). Please see video and summary Table I below for additional details.

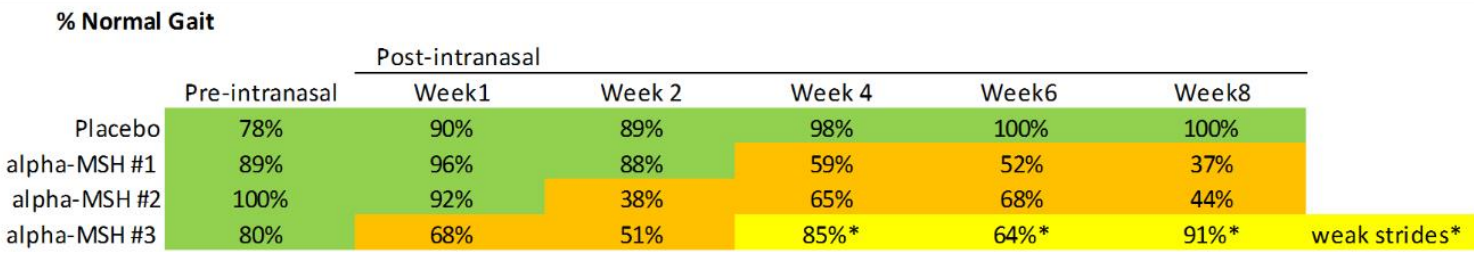

Table I: Alpha-MSH treated mice show progressive gait imbalance in a modified rotarod. \% Normal Gait is calculated by dividing the number of normal left hind leg/paw steps (where an abnormal step is when the left paw turns/moves inward to the center of gravity to compensate for right leg drag and 2 or less outer left paw digits are visible, see Figure 6, right panel) by the total number of steps taken after $1 \mathrm{~min} 30 \mathrm{secs}$. At least 3 outer left paw digits are visible in a normal step (See Figure 7, left panel).

*alpha-MSH \#3 appears with less right leg drag at Weeks 4, 6 and 8, however, the mouse also appears to have pronounced weak and sluggish strides at these times. Alpha-MSH \#1 mouse is shown in Figure 7.

Video link to PPT file: [External Data]

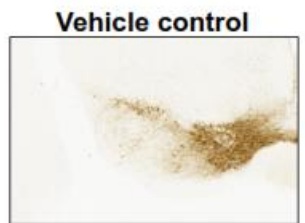

Substantia Nigra pars compacta (SNpc)
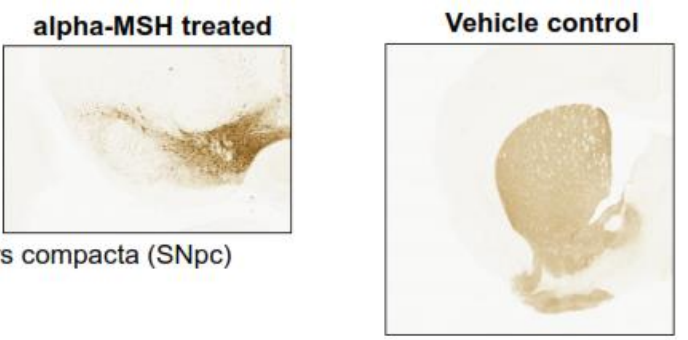

Striatum
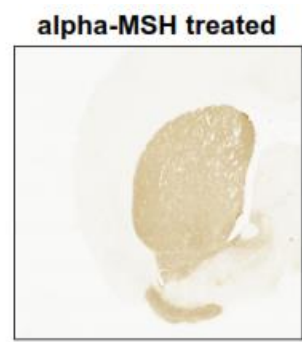

Figure 8: Alpha-MSH treated mice show very modest reduction in SNpc and striatal TH+ density. Tyrosine hydroxylase levels in the SN and striatum of mice administered alpha-MSH were examined by IHC. (Credit: NSA) 


\section{Disease induction}

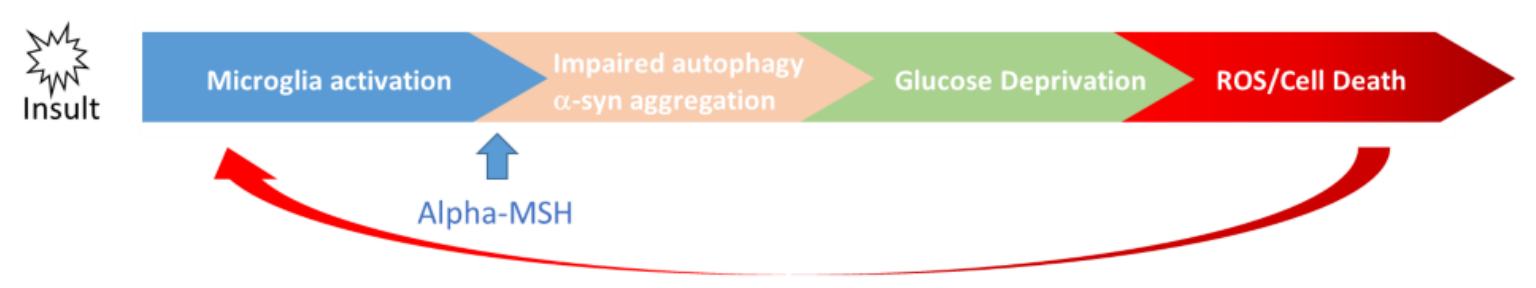

\section{Disease progression}

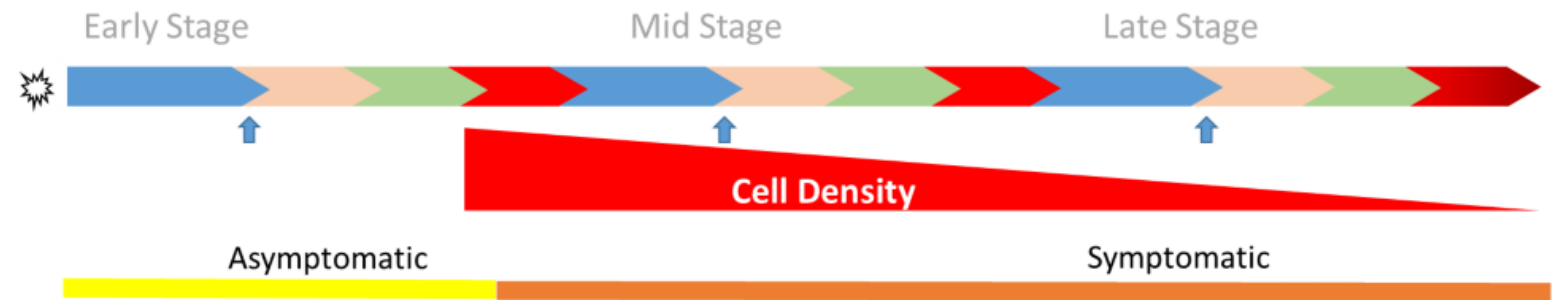

Figure 9: Proposed mechanism of PD induction and progression. Disease Induction: Exposure to abnormal levels of alpha-MSH results in impairment in cellular autophagy that give rise to accumulation and aggregation of a-syn (e.g., Lewy body). Affected neurons do not slow metabolism during low nutrient conditions and nearby healthy neurons are deprived of nutrients (e.g., glucose), starve and die to initiate another round of inflammation. Disease Progression: The cycle above is repeated many times resulting in the gradual loss of SN neurons that continues through to the later stages of the disease. 